\title{
ZUR VERFASSERSCHAFT DER BERICHTE „AUS DEUTSCHLAND” IN DER GENFER ESPÉRANCE
}

Das seinerzeit unter den Polen und Italienern ziemlich populäre Tagesblatt „L'Espérance” erschien unter der Leitung des Franzosen Armand Lévy von I 859 bis 1861 in Genf. ${ }^{1}$ Lévy $^{2}$ stand in Verbindung mit dem Prinzen Napoleon, bekannt unter den Spottnamen Plon-

1 “L'Espérance. Journal international quotidien [ab 5. August I860: Journal international]" erschien vom 25. Oktober 1859 bis 24. Februar 1861 . In-fol. Gedruckt in Genf, bei Pfeffer \& Puky. Gründer, Eigentümer und Chefredakteur: Armand Lévy (vgl. dessen Brief an den französischen Innenminister vom I3. Januar 1860; Archives nationales. Paris. $F^{18}$ 549). Redakteur: Wladyslaw Mickiewicz, Sohn des berühmten polnischen Dichters (vgl. Wladyslaw Mickiewicz, Emigracya polska I860-I 890, Krakau 1908, S. I 2). Motto der Zeitung: Patrie et Liberté. Die "Espérance" kämpfte für die nationale Befreiung der Italiener und anderer Völker, insbesondere der Polen und wollte eine Art Fortsetzung der "Tribune des peuples" sein, die Adam Mickiewicz im Jahre 1849 in Paris gegründet hatte. Vgl. W. Mickiewicz, Pamietniki, Warschau 1926-1933, 3 Bde., I, S. 309 f.; Eugène Hatin, Bibliographie historique et critique de la presse périodique française, Paris ז 866, S. 537. Die "Espérance" vertrat so stark die Sache der Polen, dass Stanislaw Zielinski (Bibliografja czasopism polskich zagranica I 830-1934, W'arschau 1935, S. 43) sie einfach als eine im Auslande erscheinende "polnische" Zeitung behandelt. Die „Espérance" schenkte auch der Arbeiterfrage viel Aufmerksamkeit; vgl. darüber D. Rjazanov, „Zur Geschichte der Ersten Internationale”, in „Marx-Engels Archiv”, r. Bd., o. J., S. I 47-1 50 .

Ich benutzte für die vorliegendeArbeit das Exemplar der,,Espérance”inderBibliothèque Publique et Universitaire, Genf, Signatur: Rc 33. Es ist unmöglich festzustellen, ob dieses Exemplar vollständig ist, denn am I4. Oktober I 860 ("Numéro du dimanche XLII") endet die Numerierung der Zeitung. Darüber hinaus enthält das soeben genannte Exemplar nur noch drei (nicht mit Ordnungszahlen versehenen) Nummern, und zwar die vom $2 \mathrm{~s}$. November 1860, 21. und 24. Februar 186I. Da die „Espérance” eine bibliographische Seltenheit ist, erwähne ich noch, dass diese drei Nummern in dem Exemplar der "Espérance", dass die Genfer Société de Lecture besitzt(Signatur: $\operatorname{Rd} 48$ ), fehlen.

2 Armand Lévy (1827-1891), französischer Publizist, Freimaurer, Freund von Adam Mickiewicz und Moses Hess, aktiver Teilnehmer an der Pariser Kommune. Über Lévy s. Jean Gaumont, Un républicain révolutionnaire romantique, Armand Lévy, in: Revuc d'histoire économique et sociale, 19. Jg., I931, S. 395-467; W. Mickiewicz, Pamietniki, passim; Roman Brandstaetter, Legjon zydowski Adama Mickiewicza(Dzieje i dokumenty), Warschau 1932, passim; Dr. Elias, Armand Lévy, un sioniste ignoré, in: Palestine, Paris, 1. Jg., Nr. 5, Februar 1928, S. 233-234; Armand Lévy (anonymer Nekrolog) in: „L'Époque”, Paris, 29. März 1891.

Die vorliegende Arbeit wurde am 31 . August 1962 in Amsterdam abgeschlossen. 
Plon und „roter" Prinz.1 Die „Espérance” war aber kein von der französischen Regierung subventioniertes Blatt. Sie nahm finanzielle Unterstützungen ausschliesslich von den gegen ihre eigenen Regierungen kämpfenden Patrioten an, ${ }^{2}$ hauptsächlich wohl von Italienern und Polen. Cavour gewährte der Zeitung keine Unterstützung, weil er sich mit Preussen und Russland nicht verfeinden wollte. ${ }^{3}$ Der Mangel an Vertrauen der französischen Regierung zur „Espérance” kommt gut zum Ausdruck in dem folgenden Dokument, das hier in extenso abgedruckt wird:

Ministère de l'Intérieur.

Paris, le 25 janvier 1860.

Services de l'Imprimerie, de la Librairie, de la Propriété littéraire, de la Presse et du Colportage.

İ̀re Section (administrative). Presse.

\section{Rapport.}

Mr. Armand Lévy, Propriétaire du journal „l'Espérance” qui se publie à Genève, demande $I^{\circ}$ l'autorisation de [le] faire circuler librement en France, $2^{\circ}$ de le faire vendre sur la voie publique.

C'est l'organe avoué des idées les plus libérales et de la cause des Nationalités.

S.A.I. le Prince Napoléon s'intéresse à Mr. Armand Lévy.

Peut-être il [sic] y aurait-il avantage à laisser cette feuille se créer à Paris des intérêts assez sérieux, pour lui commander la prudence?

On la tiendrait précisément par la faculté de lui retirer du jour au lendemain les avantages qui lui auraient été accordés."4

Durch die Verordnung vom 20. März 1860 erhielt Lévy von der französischen Regierung die Genehmigung ,à faire vendre sur la voie publique le Journal l'Espérance, publié à Genève". ${ }^{5}$ Die Zeitung

1 Vgl. Em. Hubaine, secrétaire particulier de la Maison de S.A.I. le Prince Napoléon Bonaparte, an A. Lévy, 4. Februar 1856 und 31. März 186r (Musée Adam Mickiewicz. Paris. Mss. 1035). A. Lévy an den französischen Innenminister Persigny, 22. April 1861 (Archives nationales. Paris. $F^{18}$ 549).

2 Vgl. W. Mickiewicz, Pamietniki, I, S. 365, 327.

3 Ebenda, I, S. 357.

4 Archives nationales. Paris. $\mathrm{F}^{18} 549$ (ohne Unterschrift).

5 Ebenda. 
wurde aber trotzdem von den französischen Behörden verfolgt und des öfteren konfisziert, was natürlich die Geduld der Abonnenten erschöpfte. Infolge dieser Schikanen konnte sich das Blatt schliesslich nicht behaupten. ${ }^{1}$

Lévy versuchte es zu erneuern. Am 22. April I86I wandte er sich an den Innenminister Persigny um die Genehmigung, die Zeitung in Paris zu drucken. "Mes opinions démocratiques”, schrieb er ihm, „sont connues de Votre Excellence; mais elle peut compter sur mon ardeur à combattre les ennemis actuels du gouvernement qui sont les nôtres. Car je suis convaincu que l'union loyale et forte des Napoléons et de la Démocratie peut seule épargner de nouveaux malheurs à notre patrie."2 Im Monat Mai 1861 erhielt Lévy die gewünschte Genehmigung. ${ }^{3}$ Die „Espérance” ist aber trotzdem, aus mir unbekannten Gründen, nicht wieder erschienen.

Bei der Lektüre der anonymen Korrespondenzen „aus Deutschland”, die die „Espérance” brachte, stösst man auf viele Stellen, die dem Inhalt sowie der Form nach auf Moses Hess als deren Verfasser hindeuten. Die darin entwickelten Ideen über Napoleon III., über Frankreich, Deutschland und England erinnern stark an die Gedankengänge, die man auch sonst in Hess' Schriften oft findet. Die Korrespondenzen sind von einer Franzosenliebe durchdrungen, die für Hess charakteristisch ist. Auch der dort leidenschaftlich geführte Kampf gegen die Deutschtümelei im allgemeinen und das Preussentum im besonderen sind für Hess kennzeichnend. Schliesslich ist auch das in diesen Korrespondenzen zum Ausdruck kommende jüdische Interesse ein wichtiges Element, das auf die Verfasserschaft Hessens hindeutet. ${ }^{4}$

Die „Espérance” brachte alles in allem 1 I 7 Berichte aus Deutschland, ${ }^{5}$ von denen jedoch 33 nicht als eigentliche Berichte betrachtet werden können, denn sie bestehen ausschliesslich oder beinahe ausschliesslich aus Meldungen, Erklärungen, Aufrufen etc., die anderen Zeitungen entnommen sind, so dass Anhaltspunkte für die Feststellung ihres Kompilators fehlen. Ob Hess auch einen Teil dieser 33 Stücke geliefert hat, könnte nur eine Honorarliste (oder ein

1 W. Mickiewicz, Pamietniki, I, S. 365.

2 Archives nationales. Paris. $F^{18} 549$.

3 Ebenda.

4 Vgl. z.B. „L'Espérance”, Nr. 47 vom I G. Februar I 860, Nr. 59 vom 28. Februar 1860, Nr. 68 vom 8. März I860, Nr. 107 vom 17. April I860, Nr. I 30 vom Io. Mai I860, Nr. I5 I vom I. Juni 1860 , Nr. 158 vom 8 . Juni 1860 , Nr. I6 3 vom I 3. Juni I 860 .

5 Nicht inbegriffen sind 3 Berichte, die unter dem Titel: „Nouvelles étrangères. Royaume de Prusse”, in der „Espérance" vom 30. September 1860, 21. und 24. Februar I86I erschienen sind. 
ähnliches Dokument) aufzeigen. Sachlich ist es jedoch von wenig Bedeutung, ob sie von Hess stammen oder nicht. Sieht man also von diesen 33 Beiträgen ab, so hat Hess 84 Berichte ,aus Deutschland” für die „Espérance” verfasst. ${ }^{1}$

Freilich hatte er sie nicht, wie es die „Espérance” angibt, in Berlin (oder irgendwo anders in Deutschland) geschrieben, sondern in Paris, wo er damals wohnte. Sie erschienen in den folgenden Nummern der „Espérance”:

1859: r (25. Oktober), 3, 4, ro, I 2, 16, 18, 23, 25, 28, 30, 32, 35, $37,39,40,42,43,44,46,48,50,51,55,57,59,60$;

$1860: 4,5,7,9,12,13,14,15,17,21,23,26,31,33,37,41,47,49$, $52,53,55,57,59,63,68,70,72,74,76,80,83,85,89,91,94,95,97$, IOI, 107, II3, II4, II 7, I20, I 22, I 27, I29, I30, I32, I34, I36, I4I, I48, I 50 , I I I, I $55,158,163$ (I 3. Juni). ${ }^{2}$

Ausser dem Inhalt und dem Stil der Korrespondenzen, die auf Hess' Verfasserschaft hindeuten, gibt es in den mir bekannten und zugänglichen Quellen ${ }^{3}$ auch einen externen Beweis seiner Autorschaft. Es ist dies der Brief W. Mickiewicz' in Genf an A. Lévy in Paris vom I I. Februar I 860. ${ }^{4}$ Es heisst darin:

„Il nous faut avant tout un corresp[ondant] [de] Paris et restreindre $\mathrm{H}[\mathrm{ess}]$ à l'Allemagne - $\mathrm{H}$ [ess] ne sera jamais cité nulle part pour ses corr[es]p[ondances] [de] Paris qui ne sont pas lues ni écrites et font du tort."

Hess schrieb also für die „Espérance” Korrespondenzen „aus Deutschland" im Februar 1860. Aus der Verkettung seiner Berichte ergibt sich, dass er diese auch vor und nach diesem Datum einsandte. In seinen laufenden Korrespondenzen nahm er nämlich öfters Bezug auf die vorher eingeschickten. In dem Beitrag vom 25. Mai $1860^{5} \mathrm{sagt}$

1 Nicht inbegriffen sind 4 Berichte betitelt "Autre correspondance" (Espérance, Nr. 40 vom ro. Dezember 1859, Nr. I1 3 vom 23. April 1860, Nr. I 20 vom 30. April 1860, Nr. r 27 vom 7. Mai i 860), die möglicherweise von Hess stammen.

2 Eine genaue bibliographische Erfassung dieser Berichte werde ich in einem Nachtrag zu meiner Hess-Bibliographie (The Works of Moses Hess. An Inventory of his Signed and Anonymous Publications, Manuscripts and Correspondence, Leiden 1958) vornehmen. ${ }^{3}$ Namentlich das Institut für Marxismus-Leninismus (Moskau) dürfte noch relevantes Material besitzen, das mir bei der Ausarbeitung dieser Note nicht zur Verfügung stand. 4 Der Brief (Musée Adam Mickiewicz. Paris. Mss. 1035) ist nicht unterzeichnet und ist auf der Rückseite des Briefes Alberto Redentis an Lévy (Datum: Parma, 8. Februar 1 860) geschrieben. Die Angabe, wonach der Brief von W. Mickiewicz stammt, verdanke ich der mit seiner Handschrift vertrauten Archivarin am soeben genannten Museum, Frau Irena Galezowska. Die Sichtung der Briefe an A. Lévy wurde mir dadurch exleichtert, dass Herr Marc Vuilleumier, Genf, mir den Text des Briefes vom I I. Februar 1860 freundlichst mitgeteilt hatte. Dass Lévy um diese Zeit in Paris weilte, ergibt sich aus den Adressen der an ihn gerichteten Briefe. (Musée Adam Mickiewicz. Paris. Mss. 1035, 1036).

5 Erschienen in Nr. Is I der Espérance vom I. Juni 1860. 
er, er habe „depuis plus de six mois” für die Zeitung Berichte über Deutschland erstattet. Nach meiner Berechnung dürften es damals sieben Monate gewessen sein.

Auf den Inhalt der Hess'schen Korrespondenzen, von denen mehrere von grossem Interesse sind, werde ich ein anderes Mal zurückkommen. In dieser kurzen Note kam es mir nur auf die Feststellung der Verfasserschaft an. 